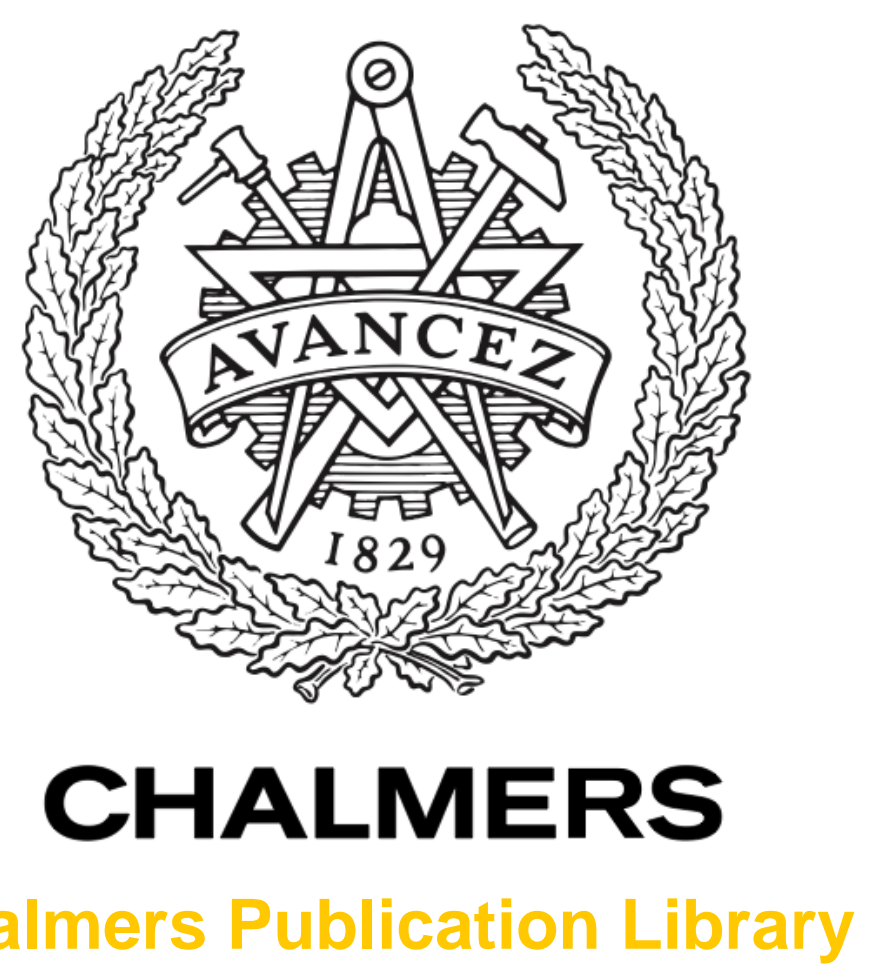

\title{
Growth mechanism of graphene on platinum: Surface catalysis and carbon segregation
}

This document has been downloaded from Chalmers Publication Library (CPL). It is the author's version of a work that was accepted for publication in:

\section{Applied Physics Letters (ISSN: 0003-6951)}

Citation for the published paper:

Sun, J. ; Nam, Y. ; Lindvall, N. (2014) "Growth mechanism of graphene on platinum:

Surface catalysis and carbon segregation". Applied Physics Letters, vol. 104(15),

http://dx.doi.org/10.1063/1.4871978

Downloaded from: http://publications.lib.chalmers.se/publication/199474

Notice: Changes introduced as a result of publishing processes such as copy-editing and formatting may not be reflected in this document. For a definitive version of this work, please refer to the published source. Please note that access to the published version might require a subscription. 


\title{
Growth mechanism of graphene on platinum: Surface catalysis and carbon segregation
}

\author{
Jie Sun, ${ }^{1, a)}$ Youngwoo Nam, ${ }^{1,2}$ Niclas Lindvall, ${ }^{1}$ Matthew T. Cole, ${ }^{3}$ Kenneth B. K. Teo, ${ }^{4}$ \\ Yung Woo Park, ${ }^{2}$ and August Yurgens ${ }^{1}$ \\ ${ }^{1}$ Quantum Device Physics Laboratory, Department of Microtechnology and Nanoscience, \\ Chalmers University of Technology, SE-41296 Gothenburg, Sweden \\ ${ }^{2}$ Department of Physics and Astronomy, Seoul National University, Seoul 151-747, South Korea \\ ${ }^{3}$ Electrical Engineering Division, Department of Engineering, University of Cambridge, \\ 9 JJ Thomson Avenue, CB3 OFA Cambridge, United Kingdom \\ ${ }^{4}$ AIXTRON Nanoinstruments Ltd., Swavesey, CB24 4FQ Cambridge, United Kingdom
}

(Received 7 October 2013; accepted 6 April 2014; published online 18 April 2014)

\begin{abstract}
A model of the graphene growth mechanism of chemical vapor deposition on platinum is proposed and verified by experiments. Surface catalysis and carbon segregation occur, respectively, at high and low temperatures in the process, representing the so-called balance and segregation regimes. Catalysis leads to self-limiting formation of large area monolayer graphene, whereas segregation results in multilayers, which evidently "grow from below." By controlling kinetic factors, dominantly monolayer graphene whose high quality has been confirmed by quantum Hall measurement can be deposited on platinum with hydrogen-rich environment, quench cooling, tiny but continuous methane flow and about $1000^{\circ} \mathrm{C}$ growth temperature. (C) 2014 AIP Publishing LLC. [http://dx.doi.org/10.1063/1.4871978]
\end{abstract}

Graphene is a layer of $s p^{2}$ carbon atoms and has shown significant potential for future nanoelectronics and nanophotonics. ${ }^{1}$ It is simultaneously conducting, transparent, and flexible, rendering it an excellent candidate for electrodes in flexible electronics. Production techniques are vital in achieving the laboratory to market transition. Recently, chemical vapor deposition (CVD) of graphene on $\mathrm{Cu}$ has proven to be largely compatible with essential semiconductor technologies, whilst also affording high material quality at a relatively low cost. Today, with the advent of roll-to-roll processing, graphene synthesis can be scaled up to $100 \mathrm{~m}$ in length. ${ }^{2}$ The commercial viability of graphene is clear, but the use of $\mathrm{Cu}$ as the ubiquitous catalyst is still somewhat contentious, with many alternatives being investigated. One example is platinum. ${ }^{3}$ An important advantage over $\mathrm{Cu}$ is the higher melting point of $\mathrm{Pt}\left(1768^{\circ} \mathrm{C}\right.$ vs. $\left.1083^{\circ} \mathrm{C}\right)$ and hence much better process robustness. That is because, with typical graphene growth temperature being $1000-1050^{\circ} \mathrm{C}, \mathrm{Cu}$ is often unintentionally molten, especially in low pressure CVD; whereas it does require super-high-precision temperature control on $\mathrm{Pt}$ since it is far from its melting point. The high price issue of $\mathrm{Pt}$ is addressed by electrochemical transfer of graphene where the Pt can be reused. ${ }^{3,4}$

Graphene growth on $\mathrm{Cu}$ is known to be principally a surface catalysis procedure due to the low carbon solubility, resulting in the so-called self-limiting formation of monolayer graphene in most cases. However, the growth mechanism of graphene on Pt has thus far not been investigated to any great extent. According to Gao et al., ${ }^{3} \mathrm{Pt}$ has a stronger catalytic ability for hydrocarbon decomposition and subsequent graphitization than $\mathrm{Cu}$. Also, $\mathrm{C}$ solubilities in $\mathrm{Pt}, \mathrm{Cu}$, and $\mathrm{Ni}$ at $1000{ }^{\circ} \mathrm{C}$ are $1.14,{ }^{5} 0.0027,{ }^{6}$ and 1.26 at. $\%,{ }^{7}$ respectively. Therefore, it is far from straightforward to understand

${ }^{\text {a)} E l e c t r o n i c ~ m a i l: ~ j i e . s u n @ c h a l m e r s . s e . ~}$ the $>98 \%$ single layer coverage in Ref. 3. Indeed, in our experiments, the deposition on Pt can easily drift to bilayer or multilayer formation regimes. Others also observed similar extensive multilayer graphene on $\mathrm{Pt}^{8}$ Nonetheless, a detailed understanding of the growth process is lacking, and it is still unclear as to whether the growth proceeds from below $^{9,10}$ or above the already formed graphene layers. ${ }^{11}$ Because little work has been done on Pt-grown graphene, ${ }^{3,8,12-14}$ we believe it is necessary to systematically investigate the growth mechanism to highlight the direction of future research. In this Letter, we ascertain that the CVD on $\mathrm{Pt}$ is a combination of high temperature surface catalysis and subsequent carbon segregation. The growth of multilayer graphene occurs from below earlier formed layers. With proper control over the growth kinetics, it is possible to achieve high quality graphene (predominately one layer) and observe quantum Hall effect reproducibly.

The polycrystalline $\mathrm{Pt}$ foil $(\sim 100 \mu \mathrm{m}$ thick, $99.99 \%)$ is purchased from GoodFellow. The graphene deposition is conducted in a cold wall low pressure CVD system ${ }^{15-17}$ (Black Magic, AIXTRON Ltd.) at $\sim 1000^{\circ} \mathrm{C}$. The catalyst is annealed for $5 \mathrm{~min}$ followed by 5-10 min of graphene growth. Gases used here are $\mathrm{CH}_{4}$ (5\% prediluted in Ar, similarly hereinafter), $\mathrm{H}_{2}$, and Ar. Finally, the finished samples are inspected using optical and scanning electron microscopy (SEM).

Fig. 1 illustrates the proposed growth mechanism. At $\sim 900^{\circ} \mathrm{C}$ and above, the hydrocarbon is cracked and subsequently forms graphene lattice catalyzed by Pt. Desorption at low pressure can affect the deposition rate. Meanwhile, the surface carbon continuously dissolves into the bulk Pt due to its high solubility. Net growth occurs when the deposition rate $>$ the absorption rate. In principle, the growth should stop once the catalyst surface is covered, rendering it a selflimiting process. Now a dynamic balance between the formation and dissolution of graphene is established (balance 


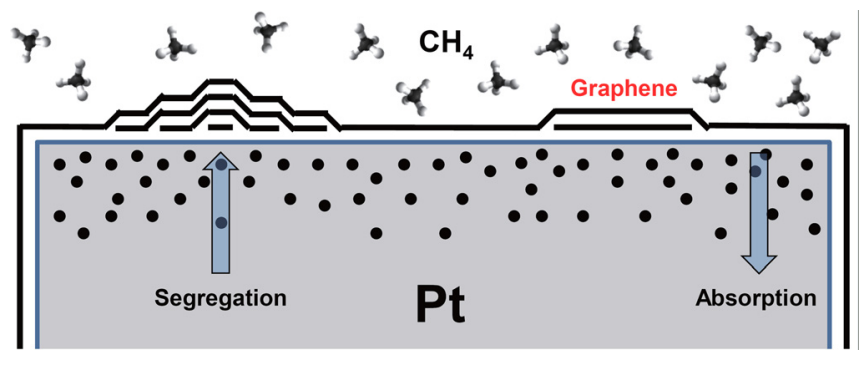

FIG. 1. Schematic illustration of the graphene growth mechanism over Pt surface. Two regimes (balance and segregation) can be defined by the relative strength of carbon deposition, absorption, and segregation.

regime, the deposition and absorption rates are equal, and segregation is negligible). In other words, some carbon atoms from the graphene lattice are absorbed into Pt and the damage is immediately healed by the new deposition. Note that if the carbon precursor is turned off too early during cooling, absorption may dominate. This can damage graphene and as such we find that it is beneficial to maintain a continuous $\mathrm{CH}_{4}$ supply. During cooling, the carbon in Pt segregates to surface due to supersaturation caused by temperature induced lowering of solubility. This suggests that multilayer graphene growth occurs via "growth from below" (segregation regime, the segregation rate $>$ the absorption rate, and deposition is very limited). The rate of segregation is directly related to the absorbed carbon into the bulk and is sensitive to cooling rate. On $\mathrm{Ni}, 600^{\circ} \mathrm{C} / \mathrm{s}$ was used to suppress multilayer formation. ${ }^{18}$ In this study, we use quench cooling $\left(1600^{\circ} \mathrm{C} / \mathrm{s}\right)$ to "freeze" the carbon atoms at high temperature. Also, it is known that a large concentration of hydrogen in CVD can hinder graphitization. ${ }^{19}$ As Pt has a much greater ability of graphene catalysis, ${ }^{3}$ it is necessary to have a $\mathrm{H}_{2}$-rich environment ${ }^{3}$ to reduce the nucleation density. This not only improves the graphene quality but also impairs the segregation, inhibiting multilayer formation. In the following discussion, we will validate this scenario based on experimental observations.

In our graphene transfer process, the Pt foils with deposited graphene are first spin-coated with poly(methyl methacrylate) (PMMA) and used as cathode in a water electrolysis cell. $\mathrm{H}_{2}$ bubbles appear at the graphene-Pt interface and delaminate the graphene/PMMA from Pt. Then, it is transferred onto $300 \mathrm{~nm} \mathrm{SiO} / / \mathrm{Si}$ and the PMMA is dissolved by acetone. Fig. 2 shows photographs taken after the transfer, summarizing several typical morphologies of Pt-grown graphene, where (a) depicts numerous multilayer regions. A close-up view (Fig. 2(b)) reveals that they have pyramid-like terraces; the thickest region can have tens of layers. Fig. 2(c) shows monolayer graphene in large area whereas (d) is a collection of very thick graphite flakes. In fact, the sample in Fig. 2(d) is grown by Joule heating ${ }^{20}$ of a Pt strip close to its melting point. Under such extreme condition, the amount of dissolved $\mathrm{C}$ is so great that severe segregation is observed, and it is almost impossible to find single layer areas.

The growth conditions clearly dictate the graphene crystal morphology. Fig. 3(a) is an SEM image of a sample grown at $1000^{\circ} \mathrm{C}$ for just $0.5 \mathrm{~min}$ (Ref. 21) with 20,20 , and $1000 \mathrm{sccm} \mathrm{CH}_{4}, \mathrm{H}_{2}$, and Ar, respectively, followed by quench cooling. While growing the sample in Fig. 3(b), the

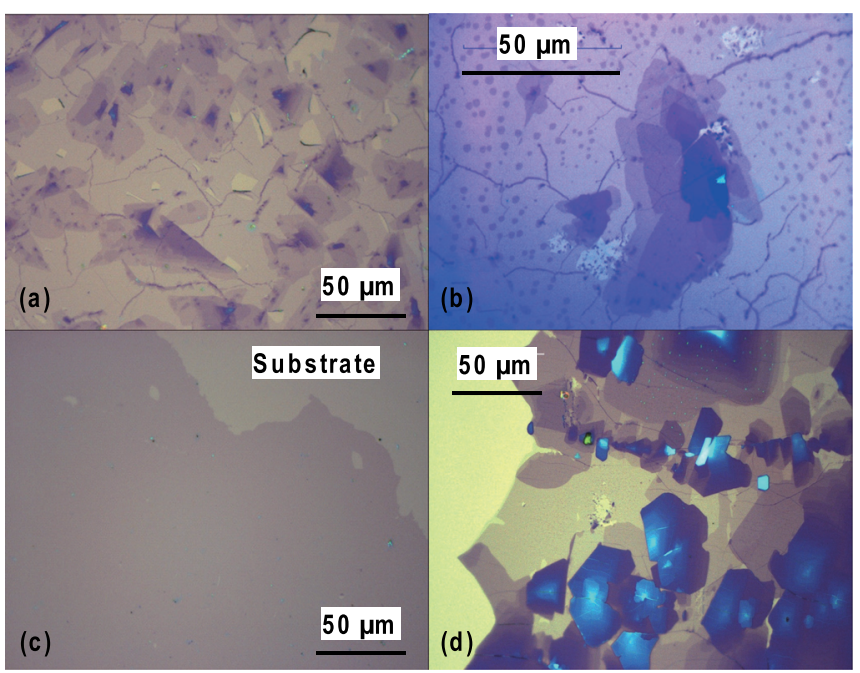

FIG. 2. Optical photos of the as-synthesized graphene after transfer to $300 \mathrm{~nm} \mathrm{SiO}_{2} / \mathrm{Si}$ substrates. (a) and (b) Typical multilayer terraces during Pt-catalyzed graphene CVD. (c) Nearly 100\% monolayer graphene (except the uncovered area). (d) Graphite crystals created due to the exceedingly high temperature.

flow rates of $\mathrm{H}_{2}$ and $\mathrm{Ar}$ are swapped (now $\mathrm{H}_{2}$-rich), and the deposition is elongated to $5 \mathrm{~min}$, whilst all other conditions are kept the same. Clearly, Fig. 3(a) demonstrates the formation of many multilayer regions, which are not detected in (b) where only the Pt grains are visible. The function of $\mathrm{H}_{2}$ for reducing multilayers is thereby confirmed. The samples in Figs. 3(c)-3(f) are all deposited at $1000^{\circ} \mathrm{C}$ for $5 \mathrm{~min}$ with 70, 1000, and $20 \mathrm{sccm} \mathrm{CH}, \mathrm{H}_{2}$, and Ar. After growth, $\mathrm{CH}_{4}$ and $\mathrm{H}_{2}$ are switched off. Quench cooling is not applied in this instance. When $T$ gets down to $900^{\circ} \mathrm{C}$, we intentionally wait for $5 \mathrm{~min}$. Afterwards, the system is gradually cooled to room temperature in argon atmosphere. Fig. 3(c) shows that

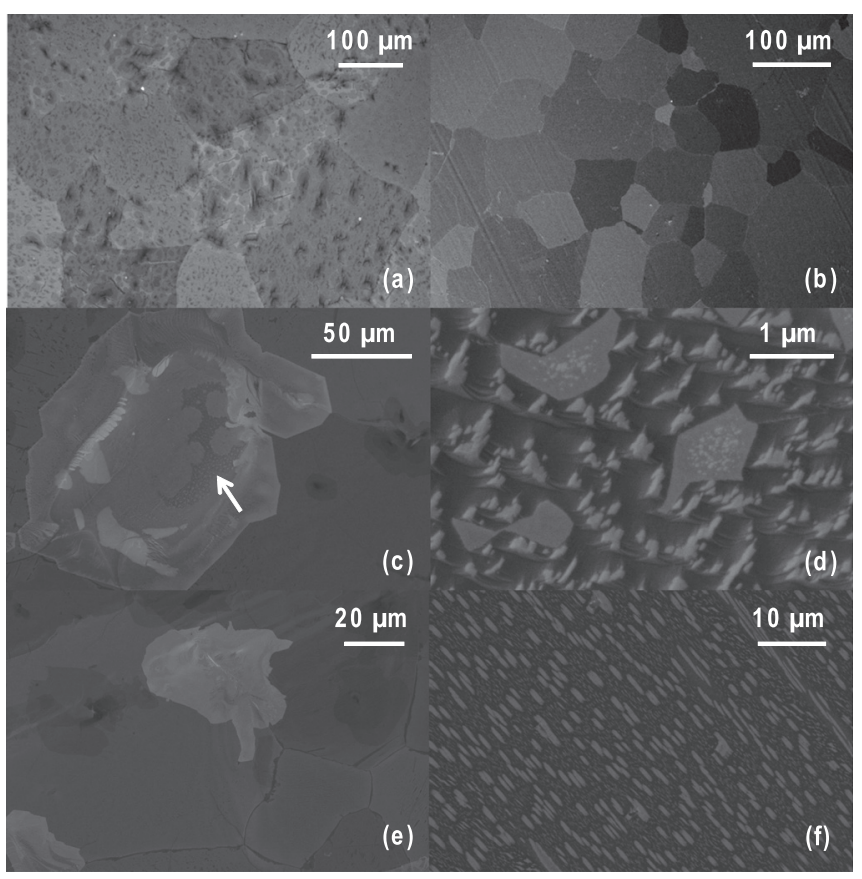

FIG. 3. SEM micrographs taken after growing graphene on Pt under different conditions. (a) and (b) Illustration of the role of $\mathrm{H}_{2}$ gas for producing dominantly single layer graphene. The samples in (c)-(f) are kept at $900^{\circ} \mathrm{C}$ for $5 \mathrm{~min}$ after the growth in order to increase the absorption of graphene into bulk Pt. 
a large area of graphene (hundreds of microns wide, indicated by the arrow) is absorbed by substrate, leaving visible only clear crystalline edges. Fig. 3(d) shows smaller graphene holes of the order of $1 \mu \mathrm{m}$ together with many nanoscale holes. From Fig. 3(e), it can be seen that in some cases the graphene absorption tends to occur more often at $\mathrm{Pt}$ grain boundaries. This is reasonable because these sites offer routes that readily facilitate $\mathrm{C}$ dissolution. In our experiments, various shapes of graphene dissolution holes such as festoons (Fig. 3(f)), long strips, and rhombus are observed, which are likely dependent on the crystal orientations of Pt grains. The message that Figs. 3(c)-3(f) conveys is that graphene absorption readily takes place at $900^{\circ} \mathrm{C}$ (and above).

Fig. 4 shows SEM micrographs taken after deposition, implying that multilayer graphene grows from below. From Fig. 4(a), it can be concluded that multilayer terraces have a tendency to appear at Pt grain boundaries, preferably at the point where three domains meet. At these apexes where it appears that more carbon has been absorbed, the carbon segregates similarly to "volcano eruption" during the cooling process. In Fig. 4(b), the subsequent graphene layers (the small islands) opt to nucleate at positions where two existing graphene domains overlap (noted by the arrow). Figs. 4(c) and 4(d) suggest that these submicron islands also nucleate at graphene wrinkles. At the growth temperature, it is reasonable to assume that graphene attaches conformally to $\mathrm{Pt}$ because it is made from individual atoms or clusters. In other words, wrinkles form at much lower $T$ due to the fact that graphene has negative thermal expansion coefficient. Since multilayer graphene nucleates at wrinkles, they must appear at low $T$ also. Thus, it is unlikely that they originate from newly decomposed $\mathrm{CH}_{4}$, but rather due to segregation from below. Another evidence is indicated by the arrows in Figs. 4(c) and 4(d), where nucleation is absent in the bilayer regions. This cannot be explained by "growth from above" because if the first layer generated a wrinkle then the second layer atop would simultaneously be wrinkled and ease the nucleation of new layers. In the "growth from below" model,

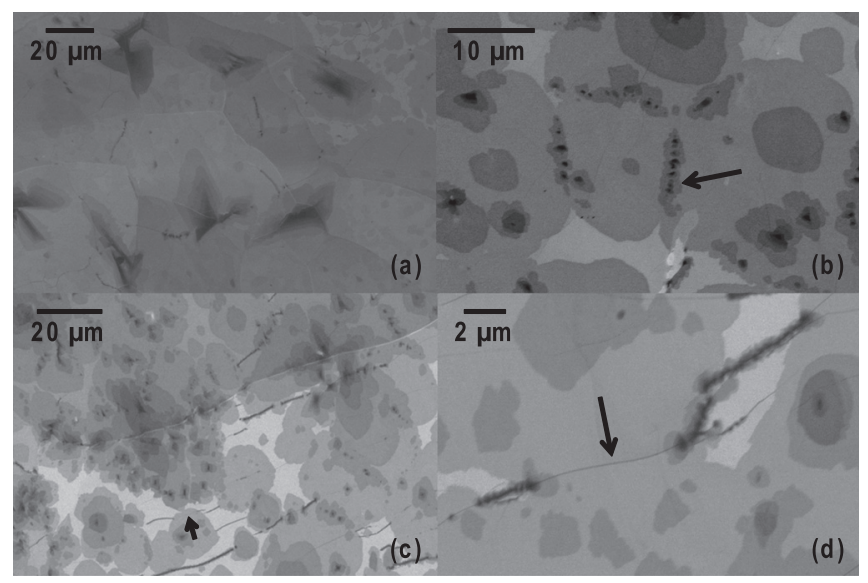

FIG. 4. SEM images of the as-deposited graphene on Pt, showing the formation of multilayers, which tend to occur at (a) Pt grain boundaries, (b) graphene flake overlaps, (c) and (d) graphene wrinkles. Nevertheless, nucleation is typically not observed near wrinkles at two layer regions (see the arrows). however, the phenomenon is natural because the second layer is conformal to the flat Pt surface and does not need to be wrinkled. Therefore, small islands of new layers will nucleate only at the first layer wrinkles that are contacting the $\mathrm{Pt}$, but not at the arrow indicated (two layer) positions.

The growth mechanism thereinbefore is in line with that on other metallic catalysts. For example, "growth from below" is also detected on $\mathrm{Rh}^{22}$ In most cases, there is an inextricable connection between the surface catalysis and carbon segregation. On $\mathrm{Ni}$, although the growth is dominantly determined by segregation, it is pointed out that surface reaction can lead to high monolayer coverage for the first $10 \mathrm{~s}$ of deposition. ${ }^{23}$ On the other hand, despite the widely believed self-limiting mechanism with $\mathrm{Cu}, \mathrm{C}$ segregation is also present in some cases. ${ }^{24}$ With these understandings, by carefully adjusting the conditions discussed previously, dominantly one layer deposition can be realized on Pt. Fig. 5 demonstrates the high quality of such graphene, which has been patterned into a Hall bar geometry by electron beam lithography (Fig. 5(a)). After metallization, at $150 \mathrm{~K}$, field effect measurements are acquired by sweeping the back gate voltage $V_{b g}$ back and forth applied to the Si substrate beneath the $300 \mathrm{~nm} \mathrm{SiO}_{2}$. Only a small hysteresis is found in the curve, and the Dirac point is close to $V_{b g}=0 \mathrm{~V}$. Fig. 5(c) plots the quantum Hall effect $\left(2 \mathrm{~K}, V_{b g}=-6 \mathrm{~V}\right)$ observed in the device, where the plateau in $R_{x y}$ is very clear and $R_{x x}$ reaches zero. The Hall mobility of holes is $\sim 2100 \mathrm{~cm}^{2} /(\mathrm{V} \mathrm{s})$ and the carrier density is $\sim 1 \times 10^{12} \mathrm{~cm}^{-2}$. We note that previously only classical Hall effect is reported in Pt-grown graphene. Fig. 5(d) is a representative Raman spectrum measured in the graphene, showing a narrow 2D peak $\left(\sim 40 \mathrm{~cm}^{-1}\right)$ with a $2 \mathrm{D}$-to-G ratio of $\sim 2$. The carrier mean free path (in $\mathrm{nm}$ ) can be estimated from the average distance between defects in the graphene by ${ }^{25} l=\lambda^{2} \sqrt{1.8 \times 10^{-9}\left(I_{G} / I_{D}\right)}=24.2 \mathrm{~nm}$, where $I_{G}$ and $I_{D}$ are intensities of Raman $\mathrm{G}$ and D peaks, respectively, and $\lambda$ is the wavenumber of the excitation laser in $\mathrm{nm}(514 \mathrm{~nm}$ in our case). Hence, the mobility can be
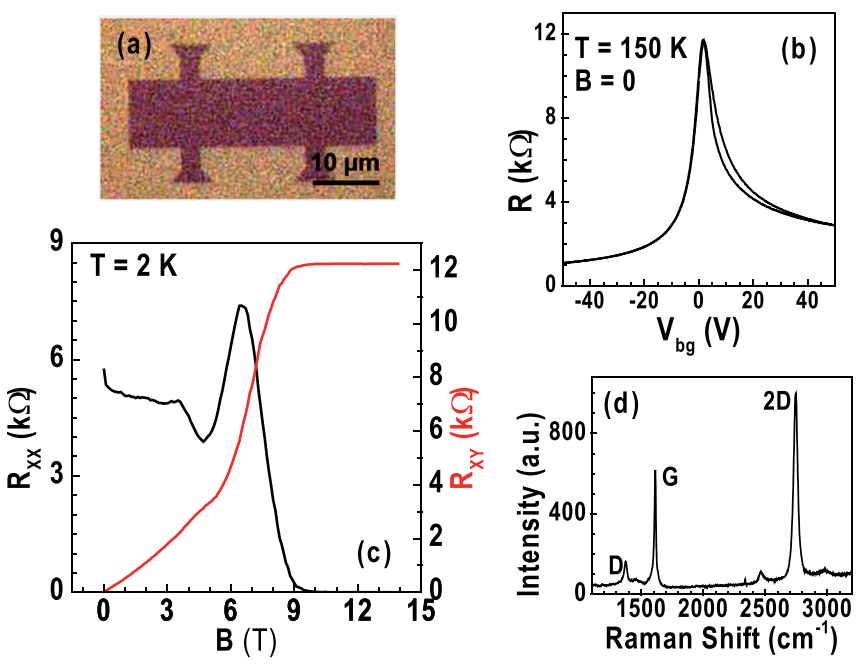

FIG. 5. (a) Optical image of the graphene Hall-bar structured used in the measurement. (b) Electric field effect measured in the graphene by sweeping the back gate voltage at $150 \mathrm{~K}$. (c) Quantum Hall effect measured at $2 \mathrm{~K}$ showing a clear plateau. (d) Typical Raman spectrum of the graphene transferred to $\mathrm{SiO}_{2} / \mathrm{Si}$. 
calculated by ${ }^{26} \mu=2 e l / h \sqrt{\pi / n}$ (in SI units, where $n$ is carrier density) to be $0.21 \mathrm{~m}^{2} /(\mathrm{V} \mathrm{s})$, in good agreement with the electrical measurement.

In summary, large area graphene has been grown on platinum by CVD. A model elucidating the growth mechanism is proposed in terms of surface catalysis at high temperature and carbon segregation during cooling down. There are primarily three physical processes taking place, namely, graphene deposition, absorption, and segregation, which divide the CVD into balance and segregation regimes. Multilayer graphene grows from below already deposited layers. The picture agrees well with the experiments and can be used to fine tune the growth parameters to obtain high quality monolayer graphene by using $\mathrm{H}_{2}$, quench cooling, small but continuous hydrocarbon flow, and intermediate growth temperature. As a result, quantum Hall effect is observed in Pt-catalyzed monolayer graphene.

This work was funded by Swedish Research Council, Swedish Foundation for Strategic Research, Stiftelsen Olle Engkvist Byggmästare, Chalmers Area of Advance Nano, and Knut and Alice Wallenberg Foundation. Also the Leading Foreign Research Institutes Recruitment Program (200900514) of NRF, Korea, is gratefully acknowledged. M. T. Cole thanks Winston Churchill Trust for financial support. K. B. K. Teo acknowledges the support of EU project GRAFOL.

${ }^{1}$ K. S. Novoselov, V. I. Fal'ko, L. Colombo, P. R. Gellert, M. G. Schwab, and K. Kim, Nature 490, 192 (2012).

${ }^{2}$ T. Kobayashi, M. Bando, N. Kimura, K. Shimizu, K. Kadono, N. Umezu, K. Miyahara, S. Hayazaki, S. Nagal, Y. Mizuguchi, Y. Murakami, and D. Hobara, Appl. Phys. Lett. 102, 023112 (2013).

${ }^{3}$ L. Gao, W. Ren, H. Xu, L. Jin, Z. Wang, T. Ma, L.-P. Ma, Z. Zhang, Q. Fu, L.-M. Peng, X. Bao, and H.-M. Cheng, Nat. Commun. 3, 669 (2012).

${ }^{4}$ C. J. L. de la Rosa, J. Sun, N. Lindvall, M. T. Cole, Y. Nam, M. Löffler, E. Olsson, K. B. K. Teo, and A. Yurgens, Appl. Phys. Lett. 102, 022101 (2013).
${ }^{5}$ R. H. Siller, W. A. Oates, and R. B. McLellan, J. Less-Common Met. 16, 71 (1968).

${ }^{6}$ R. B. McLellan, Scr. Metall. 3, 389 (1969).

${ }^{7}$ B. Longson and A. W. Thorley, J. Appl. Chem. 20, 372 (1970).

${ }^{8}$ J. Ping and M. S. Fuhrer, Nano Lett. 12, 4635 (2012).

${ }^{9}$ S. Nie, W. Wu, S. Xing, Q. Yu, J. Bao, S. Pei, and K. F. McCarty, New J. Phys. 14, 093028 (2012).

${ }^{10}$ Q. Li, H. Chou, J.-H. Zhong, J.-Y. Liu, A. Dolocan, J. Zhang, Y. Zhou, R. S. Ruoff, S. Chen, and W. Cai, Nano Lett. 13, 486 (2013).

${ }^{11}$ L. Liu, H. Zhou, R. Cheng, W. J. Yu, Y. Liu, Y. Chen, J. Shaw, X. Zhong, Y. Huang, and X. Duan, ACS Nano 6, 8241 (2012).

${ }^{12}$ P. Sutter, J. T. Sadowski, and E. Sutter, Phys. Rev. B 80, 245411 (2009).

${ }^{13}$ Y. Nam, J. Sun, N. Lindvall, S. J. Yang, D. Kireev, C. R. Park, Y. W. Park, and A. Yurgens, Appl. Phys. Lett. 103, 233110 (2013).

${ }^{14}$ R. Shi, H. Xu, B. Chen, Z. Zhang, and L.-M. Peng, Appl. Phys. Lett. 102, $113102(2013)$.

${ }^{15}$ J. Sun, N. Lindvall, M. T. Cole, K. T. T. Angel, T. Wang, K. B. K. Teo, D. H. C. Chua, J. Liu, and A. Yurgens, IEEE Trans. Nanotechnol. 11, 255 (2012).

${ }^{16}$ J. Sun, N. Lindvall, M. T. Cole, K. B. K. Teo, and A. Yurgens, Appl. Phys. Lett. 98, 252107 (2011).

${ }^{17}$ J. Sun, M. T. Cole, N. Lindvall, K. B. K. Teo, and A. Yurgens, Appl. Phys. Lett. 100, 022102 (2012).

${ }^{18}$ K. S. Kim, Y. Zhao, H. Jang, S. Y. Lee, J. M. Kim, K. S. Kim, J.-H. Ahn, P. Kim, J.-Y. Choi, and B. H. Hong, Nature 457, 706 (2009).

${ }^{19}$ Y. Zhang, Z. Li, P. Kim, L. Zhang, and C. Zhou, ACS Nano 6, 126 (2012).

${ }^{20}$ Electrical current is directly sent through the Pt foil in the CVD chamber during growth. We do not know the exact temperature value because our thermal couple can only measure up to $1000^{\circ} \mathrm{C}$. It can be empirically estimated to be $\sim 1500^{\circ} \mathrm{C}$ as a small additional increase in the power will result in Pt melting.

${ }^{21}$ In this Ar-rich atmosphere, even longer growth time will deposit too much carbon on the Pt surface.

${ }^{22}$ M. Liu, Y. Gao, Y. Zhang, Y. Zhang, D. Ma, Q. Ji, T. Gao, Y. Chen, and Z. Liu, Small 9, 1360 (2013).

${ }^{23}$ L. Huang, Q. H. Chang, G. L. Guo, Y. Liu, Y. Q. Xie, T. Wang, B. Ling, and H. F. Yang, Carbon 50, 551 (2012).

${ }^{24} \mathrm{H}$. Bi, F. Huang, W. Zhao, X. Lu, J. Chen, T. Lin, D. Wan, X. Xie, and M. Jiang, Carbon 50, 2703 (2012).

${ }^{25}$ A. C. Ferrari and D. M. Basko, Nat. Nanotechnol. 8, 235 (2013).

${ }^{26}$ A. S. Mayorov, R. V. Gorbachev, S. V. Morozov, L. Britnell, R. Jalil, L. A. Ponomarenko, P. Blake, K. S. Novoselov, K. Watanabe, T. Taniguchi, and A. K. Geim, Nano Lett. 11, 2396 (2011). 